\title{
Developing enterprise sponsored virtual communities: the case of a SME's knowledge community
}

\author{
António Lucas Soares ${ }^{1}$, Dora Simões ${ }^{1}$, Manuel Silva $^{1}$, Ricardo Madureira $^{1}$ \\ ${ }^{1}$ INESC Porto, Rua Roberto Frias, Campus da FEUP, 4200 Porto, Portugal \\ asoares@inescporto,dsp@isca.ua.pt, mdasilva@iscap.ipp.pt, \\ ricardo.madureira@inescporto.pt
}

\begin{abstract}
This paper presents a case in the development of a knowledge community support system in the context of an industrial association group in the construction sector. This system is a result of the Know-Construct project which aims at providing association sponsored SME communities of the construction sector with a sophisticated information management platform and community building tools for knowledge sharing. The paper begins by characterizing the so-called construction industry knowledge community. The Know-Construct system concept and the its general architecture are described, focusing on the semantic resources, in particular the ontologies structure. The final part of the paper depicts the approach to the actual introduction of the system in the community. An action-research approach was planned to obtain research results regarding the social acceptance of semantic resources such as the ontologies and technical classifications used in system.
\end{abstract}

Keywords: enterprise sponsored virtual communities, ontology based information systems, action-research

\section{Introduction}

Enterprise sponsored virtual communities (ESVC) are emerging as serious business schemes fostering collaboration and knowledge sharing both intra and interorganizations. The community paradigm is winning space among more established inter-organizational interaction forms such as chains or networks, complementing them in some cases. ESVCs are complex socio-technical systems, difficult to design and maintain, needing multi-disciplinary approaches for their development. This paper ${ }^{1}$ presents a case in the development of a knowledge community support (KCS) system in the context of an Industrial Association Group (IAG) in the construction sector. The system is a result of the Know-Construct project which aims at providing IAGs sponsored SME communities of the construction sector with a sophisticated information management platform and community building tools for knowledge sharing. The Know-Construct project ${ }^{2}$ intends to improve the effectiveness of the

1 Part of this paper result from research work financed by the Fundação para a Ciência e Tecnologia MCTES - POCTI/GES/49202

2 COLL-CT-2004-500276 KNOW-CONSTRUCT Internet Platform for Knowledge-based Customer Needs Management and Collaboration among SMEs (2005-2007). Project co-funded by the European Community. "Horizontal Research Activities Involving SMEs-Collective Research Programme. 
Construction Industry (CI) SME's by improving and extending the relationship with their customers through an innovative support regarding information and knowledge about products, processes and associated issues. This is achieved through specifically developed tools, supporting in particular the formation and operation of SME's knowledge communities in the context of Industry Association Groups (IAG). More specifically, these objectives aim (i) to provide a platform to support the creation and management of a community of CI SME's, coordinated by an association, fostering collaboration and knowledge sharing among its members (shareable knowledge includes, besides product and services information, companies' experience, e.g. best practices); and (ii) to provide problem-solving support to the individual IAG member's customers regarding the products' selection, applications and processes, as well as addressing other related problems such as legislative issues, safety issues, etc. This leads to a wider and deeper technical and professional competence shared by the SME's community, fundamental to its ability to satisfy customer needs, obtained through closer co-operation and knowledge exchange. It will be materialized as an internet-based platform that will offer the possibility to establish a "one to one" communications medium. Manufacturers and wholesalers (SME) may interact with their customers, advising them on specific topics relying also on knowledge created and maintained by the community of SME's mentioned previously.

These objectives where translated into two main modules of the $\mathrm{KC}$ system: (i) Customer Needs Management (CNM) System: a decision making support system regarding the products characteristics, applications and other consultancy services for SMEs customers applying a "web enabled dialogue"; and (ii) Knowledge Community Support (KCS) System: a system for SMEs to support a form of co-operation through the creation of Knowledge Communities of SMEs in Construction Industry.

\section{Characterization of the CIK community}

There are many explanations around the community concept. After thorough review of the literature on this subject, a characterization of the Construction Industry Knowledge (CIK) community according to three approaches was made: type of utility, participant's behavior and typology.

The classification of different types of utility [1] presents a basic predictive model of different communities types, with particular relevance for those able to generate some type of utility for someone. The CIK Community can be classified as an hybrid of a Practice and Interest Community. On the one hand, company employees as individuals should see a direct utility to their particular jobs when participating in the CIK community. On the other hand this direct utility also comes into light when an employee (and consequently the company) realizes that, when solving a problem for an important customer, the information/knowledge used to reach the solution has been made available by other community members. Nevertheless, not all the activities can be tracked to a causal benefit to the SME. For example, a chat session between two employees exchanging professional experiences or a report on a concern regarding the performance of a material in a news or blog entry by another employee, are activities that make sense in a community but cannot be assigned a concrete and immediate value for the organization. 
Looking at professional development as the process of continually developing knowledge, skills and attitudes of professionals by means of formal and informal learning in the course of practice, the use of on-line knowledge communities for this purpose implies that an on-line knowledge community has to support this process. As a CIK community member, professionals in the construction sector will have a place for continual professional development that gives: individualized, flexible and easy access to a coherent and up to date knowledge domain, a range of opportunities to interact with like-minded persons and a range of opportunities to develop and exploit the knowledge domain. An example of this is: applying knowledge, learning from it, guiding others, disseminating ideas and results or doing research, embedded in a professional network. Our premise is that the membership of professionals of an online knowledge community will have positive effects on their continuing development, expressed not only in competences like knowledge, skills, experiences and attitude, but also, in the acquisition of organizational knowledge assets expressed in the growth and elaboration of professional knowledge, applicability of knowledge and legitimacy of knowledge. Based on work of [2], the CIK Community can be further characterized: (i) the goal is to develop and exploit knowledge about civil construction sector; (ii) there are continuous interactions between participants to meet these goals, (iii) information and communication processes are continuously made explicit, (iv) it adds value to the participants (professionals within the sector and customers alike), the on-line meeting place that is usable, (v) the culture focuses on the participants' needs as the route to high performance; involvement and participation create a sense of responsibility and ownership and, hence, greater commitment, and (vi) the context is highly complex and constantly evolving and the CIK Community will have to continuously cope with the expectations of its participants and their context of use of the system.

Based on the typology of virtual communities proposed by Porter [3] where the communities are classified under two levels - establishment and relationship orientation - the CIK Community is classified as an organization-sponsored community relatively to type of establishment and as a commercial community relatively to the relationship orientation. This community will have key stakeholders and/or beneficiaries (e.g. customers) that will play an important part in sponsoring the community's mission and goals. Being an organization-sponsored community, it will foster relationships both among members (e.g. professionals belonging to the associations of the project partnership) and between individual members (e.g. customers) and the sponsoring organizations (associations of the project partnership). Based on the classification of the CIK Community under the virtual community concept and the attributes commonly suggested in the literature to characterize virtual communities [4], the key attributes of the CIK Community. The key attributes that characterize of the CIK Community can be summarized as the Five Ps [3]: Purpose (Content of Interaction), Place (Extent of Technology Mediation of Interaction), Platform (Design of Interaction), Population (Pattern of Interaction) and Profit Model (Return on Interaction) (see Figure 1). 

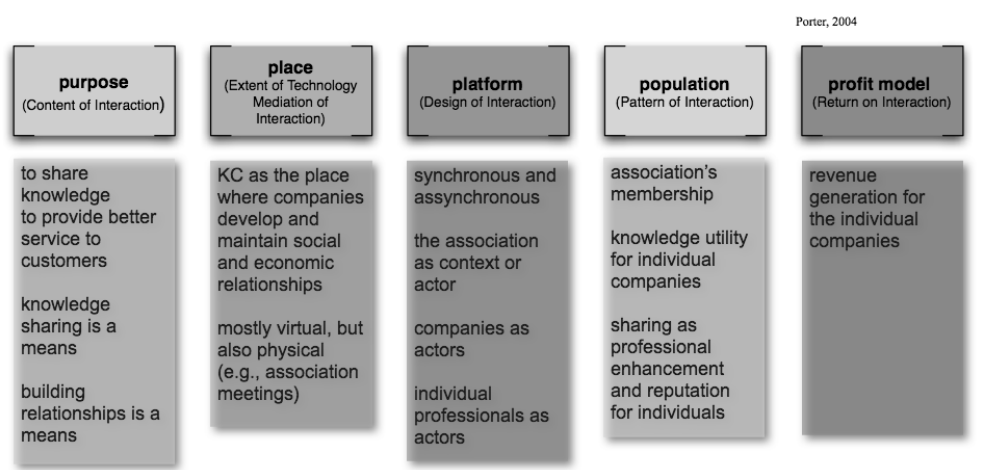

Figure 1 - Characterization of the CIK community according to the five Ps [3]

\section{The KCS System Concept}

\subsection{The KCS System Functional Architecture}

As mentioned before, the $\mathrm{KC}$ project has a very specific goal: to enable individual SME's to better solve their customers' problems. Therefore, KCS is focused on pursuing this goal in the first place. Although a knowledge community encompasses, as stated before, mechanisms that surpass this simple instrumental goal, the initial vision of the KCS system was specifically conceived having this in mind. This means that the KCS system supports CIK community building in a broad sense, though focused fundamentally in generating wide ranging and detailed knowledge to be used in managing the SME's customers' relationship, particularly in problem solving. The operationalization of KCS system is made through the use of mechanisms that will allow [5]: (i) the support of social processes (trust building, group formation and coordination), i.e., conditions for tacit knowledge exchange; (ii) increased levels of interactivity and to stimulate the dynamic exchange of knowledge (collaborative content management systems); and (iii) support to the personalization of user interaction (via the selection and presentation of content), maximize the impact of distributed knowledge and also facilitate the establishment of new relationships between the users. Keeping in mind the basic idea that the KCS system should support the CIK Community building in a broad sense, though focused primarily in generating a knowledge base that is as comprehensive and detailed as possible so as to be used in managing the SME's customers' relationship, particularly in problem solving, the following general functions of this module were specified.

Community building tools that support the processes of community building by providing the instruments to foster professional interaction and socialization; forums and weblogs are two such instruments and are tailored in KCS to be tightly integrated with the semantic structure supporting knowledge management in KC; Semantic resources management i.e., the infrastructure and corresponding set of functionalities that support information and knowledge acquisition, organization and storage in KCS system, enabling (i) the management of classifications, thesauri and 
vocabulary, (ii) the acquisition of knowledge from digital content (including forums and weblogs entries, web pages, etc.) both internal to the CIK and from external sources, (iii) the maintenance of an ontology which is the base of knowledge representation, access and storage; Knowledge resources access i.e., creating, searching and updating knowledge resources constitutes a fundamental set of functionalities in KCS; although much of the community's information/knowledge will be created in communication/interaction processes (forums, weblogs), there will be also the need to create/access knowledge in a more structured way; digital content management and document management are the natural approaches regarding this issue.

This generic architecture can be decomposed in two layers (see Figure 2): KCS Core Services layer and Systems/Applications layer. KCS Core Services layer provides a set of services centred in the semantic resources management of $\mathrm{KC}$. The basic architectural idea of KCS is to have a set of services to be used by specific, adaptable and, eventually, off-the-shelf systems/applications. The rationale is to take advantage of as great a number of open source systems/applications as possible that already provide the end user functionalities required in a knowledge community. For example, we can use a content management system (CMS) such as Zope/Plone or OpenCMS providing off-the-shelf functionalities to organize reports, data sheets, legal documents, and to publish web pages related with some community topic.

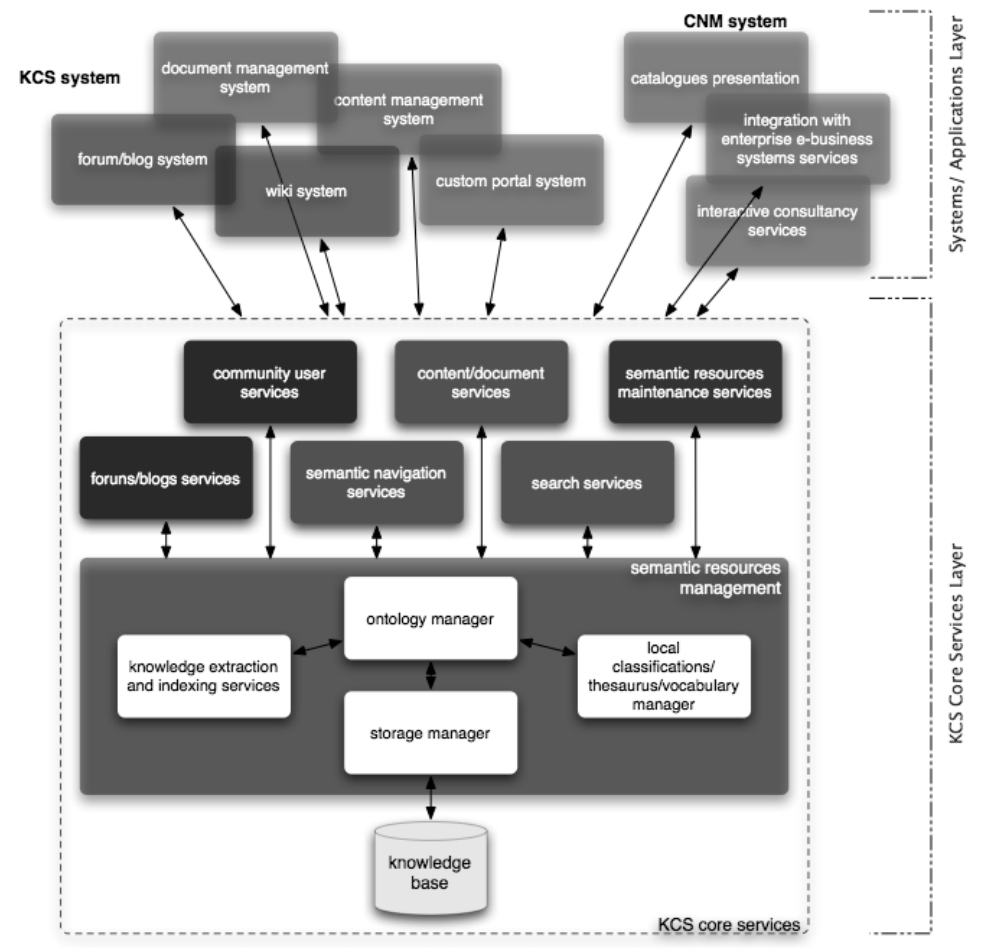

Figure 2 - Knowledge Community Support system 
The CMS is configurable and extendeable to use content/document services, search services and semantic navigation services in order to provide value added knowledge management to the community.

\subsection{Ontologies Structure and Content}

During the requirements analysis phase, a perception gained strength - to deal with the very concrete reality of the SMEs of each country and each Construction Industry Knowledge (CIK) Community of $\mathrm{KCS}$, the $\mathrm{KC}$ system need to include a local ontology that would answer the KC partners' particular professional and cultural needs and attend to its social context of use. This meant the need to construct not one, but more than one ontology. Different countries (or even different associations) need to use local classifications, thesauri or ontologies. As different countries have different local ontologies, the best process to "plug-in" a local ontology into the CIK ontology is to align both ontologies. This has the advantage of making it easier to maintain and evolve the ontologies and, in practical terms, the option for ontologies integration can be a lot more difficult when managing the ontologies and its evolution process, for example when discovering the part of the integrated ontology that will suffer changes. The central ontology (CIK ontology) reflects standards and related classification schemes in the industry and the local ontologies will account for the individualized SME conceptual schemes, i.e. they will be strongly related to the consortium partners' needs.

The CIK Ontology that defines the domain of the CI summarized in the following sentence: The Construction Industry involves a set of resources that follow certain conditions which are used or required in a process that leads to results. As such, the proposed taxonomy includes four domains to classify these major concepts: Construction Resource; Construction Process; Construction Result; Technical Topic. The first three domains coincide with the major themes in the ISO 12006-2 standard [6]. The other domain (Technical Topic) is the result of the integration of an eCOGNOS module, further developed so as to include issues related to the CI that are not covered by the e-COGNOS ontology and IFC model. All domains integrate modules from e-Cognos ontology [7].

\section{Implementing the KCS system in an IAG sponsored community}

Empirical studies regarding the company's adoption and use of information systems based on advanced semantic infrastructures are scarce in the literature. Particularly when it comes to ESVC, our knowledge about the context, conditions and actual use of such type of systems is almost inexistent (as much as the authors are aware). In this project we decided to go further in the development of the Know-Construct system, taking advantage of prototype testing in realistic organizational situations to research the adoption and use of semantic enabled information systems in ESVCs. In this section we will describe this approach emphasizing the research design and planning. This research is undergoing which means that existing results will be object of a future paper. 


\subsection{An action research approach}

Action research encompasses several different approaches. In this respect, Coghlan [8] distinguishes between mechanistic and organistic approaches. In addition, there are specific elaborations according to the type of inquiry, namely clinical, developmental, appreciative, cooperative, and participatory. These several ways of conducting action research may also reflect an emphasis on either the practical outcome [9] or the inquiry process [10]. In terms of the inquiry process, Avison [11] identifies problem diagnosis, change and reflection as the core activities of action research. Coghlan [12] similarly refers to planning, taking action, evaluating action and further planning. For the specific case of information systems research, Iversen [13] refers to initiating, iterating and closing, based on specific tasks that we synthesize below: 1 . Establish the research team; 2 . Identify the problem situation; 3 . Delimitate the theoretical context; 4. Identify the research questions; 5. Plan specific tasks and coordination mechanisms; 6. Implement specific tasks and mechanisms; 7. Evaluate research and problem-solving; 8. Repeat cycle 5 to 7 as required to answer 2 and $4 ; 9$. Exit cycle 5 to $7 ; 10$. Elicit theoretical, empirical and methodological results. In practice, the action research's main dilemma is between researchers' control and participation. Sense [14], for instance, defines control as "the desire/actions of the researcher to influence the research proceedings in ways that 'drive' the process along the researcher's pre-determined path"; and participation as "the active engagement of the researcher in the activities of the study but without the need to dictate/mandate the processes in play". According to Sense [14] this dilemma can be attenuated by clarifying the roles of the participants and respective expectations while adjusting to changes in the project such as the mix of team members, the social, political and personal motivations, and the exact research questions and problem situation. In our view, this dilemma between control and participation can be further attenuated by ensuring a consensus on what constitutes the content (research questions and problem situation) and the context (theoretical and empirical) of the project. In other words, although the content and context of the study may change over time, the research team may still agree on the next task ahead. It is this permanent consensus that ultimately ensures the viability of action research.

\subsection{Designing the action-research based approach}

A first technical prototype of the KCS system was developed in two modules: (i) a minimally configured Content Management System providing basic content management, and basic community building functionalities i.e., a forum and a weblog application, and (ii) semantic functionalities module providing classification and ontology browsing, semantic searching, and content annotation. Two IAGs were selected to implement the first socio-technical KC prototype. Besides the IAG management unit, six companies in each IAG were chosen for the prototype testing and study. In each of these companies a set of people was selected to form the KC testing groups. During a training day they were introduced to the CIK community concept and to the use of the KCS system. 
Table 1. Specific topics to research through the KC prototype testing

\begin{tabular}{|c|c|}
\hline semantic resources related & ESVC related \\
\hline $\begin{array}{l}\text { cognitive apprehension of the } \\
\text { ontology and classification schemes }\end{array}$ & $\begin{array}{l}\text { use of content shared within the } \\
\text { community; trust and perceived } \\
\text { importance of the information }\end{array}$ \\
\hline $\begin{array}{l}\text { intended and effective use of the } \\
\text { ontology }\end{array}$ & $\begin{array}{l}\text { intention to share information } \\
\text { according a "community" worldview; } \\
\text { actual uploading of content; quality of } \\
\text { the shared information }\end{array}$ \\
\hline $\begin{array}{l}\text { contextual factors of ontology } \\
\text { adoption: country, type of } \\
\text { association, type of company }\end{array}$ & $\begin{array}{l}\text { intention to use community building } \\
\text { tools (forums, weblogs, wikis); actual } \\
\text { use of community building tools }\end{array}$ \\
\hline
\end{tabular}

The general research questions can be formulated as the following: (i) how do workers in SMEs adopt and accept collaborative information management strategies, and (ii) how do workers belonging to SMEs participating in some form of network apprehend and appropriate the concept of enterprise sponsored virtual community. These research questions can be detailed in more specific topics (see Table 1).

\subsection{Structure and process of the action-research intervention}

Following the general guidelines from section 4.1 and the research questions formulated in section 4.2 the structure and process of the socio-technical prototype testing was designed, being depicted in the following figure:

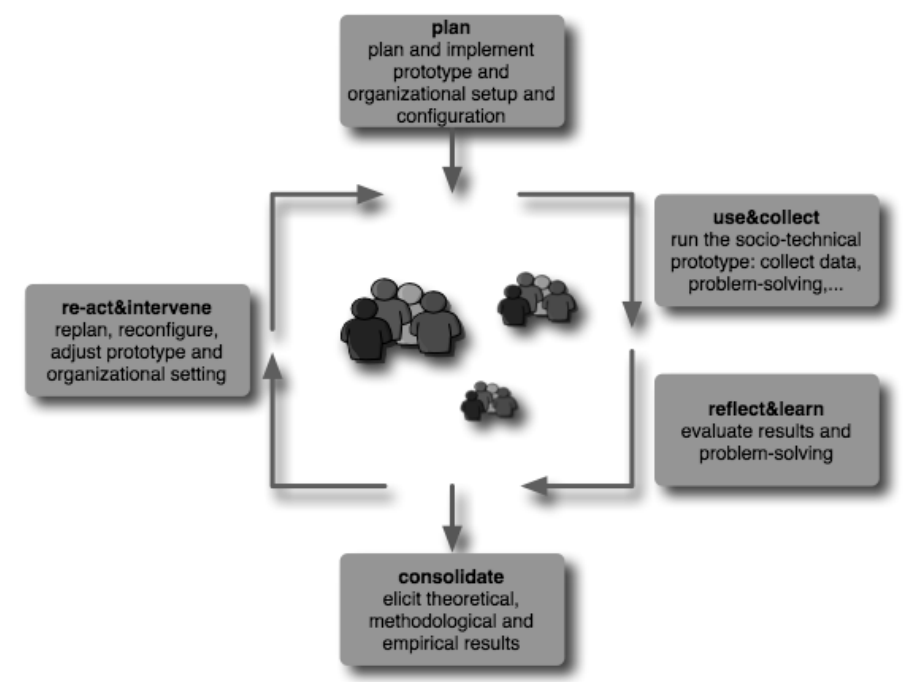

Figure 3 - The action research process of the Know-Construct prototype testing 


\section{Conclusions and further work}

This paper presented the main issues in the development of a system to support a knowledge community of SME's in the Construction Industry. We presented the characterization of the Construct Industry Knowledge Community according to three approaches in the context of the Know-Construct project. Summarizing, the CIK Community was conceptualized as an aggregation of professionals and customers who interact around a specific shared interest of construction sector, sharing information and knowledge about products, services, techniques, legal aspects, experiences, etc, and where the interaction is totally supported and/or mediated by web technology and guided by some agreed protocols or norms.

To present the generic architecture of the KCS (Knowledge Community Support) system that supports the CIK community, the concept, layers and high-level design of KCS system were described. The high-level design organizes KC functionalities in four main areas: modelling (ontology development and use), tagging, query, and visualization. The future work will involve, obviously, the implementation and validation of the system. Further, one of the crucial development and validation tasks is related to the ontology implementation and the associated functionalities.

Given the selection, evaluation and structuring process described in this paper, it is highly probable that the CIK ontology reflects the standards and related classification schemes in the industry on the one hand and, on the other, that the local more specific ontologies will account for the individualized SME conceptual schemes, i.e. they will strongly relate to consortium partners' needs, as identified in the analysis of business case scenarios and in the users' requirement definitions.

The implemented method aims at developing a methodology of common Construction Industry Knowledge representation applicable to large sets of SMEs in the construction industry as a basis for the establishment of a knowledge community. Because of the available time frame, the described method was not as fine grained as desired. Therefore, further work in this area will be directed to detail the evaluation criteria. The next steps will involve the definition of the ontologies and maintenance strategies.

Although the phase regarding the analysis and specification of the KCS system (basic functionalities, semantic resources definition: high-level ontology and local ontologies definition, integration processes) has been comprehensive, and involved the users [15], we concluded that it was necessary to refine the requirements and design options through the use of a socio-technical prototype. This process is complex as we are dealing with virtual communities. Thus, there was the need of using innovative ways to establish a social test environment in order to achieve the goals of prototyping. We presented the design and planning for an action-research based prototype testing, which means not only to obtain new knowledge about the use of advanced semantic based tools for community support but also an innovation for the companies participating in the project. 


\section{References}

1. Cornejo, M.: Utility, value and Knowledge Communities. Knowledge Communities. online:http://www.providersedge.com/docs/km_articles/Utility_Value_and_K-

Communities.pdf , (2003). (last accessed January 2006)

2. De Vries, S. and P. Kommers: "Online knowledge communities: future trends and research issues." International Journal of Web Based Communities (1): (2004) 115 - 123.

3. Porter, C. E.: "A Typology of Virtual Communities: A Multi-Disciplinary Foundation for Future Research." Journal of Computer-Mediated Communication (JCMC) 10 (1), (2004) Article 3.

4. Blanchard, A.: Virtual behaviour settings: An application of behaviour setting theories to virtual communities. Journal of Computer Mediated Communication, 9(2) (2004).

5. Nabeth, T., Angehrn A. A.: Towards Personalized, Socially Aware and Active Knowledge Management Systems. E-business and E-work - Challenges and Achievements in E-business and E-work, Amsterdam, Holland, IOS Press (2002)

6. ISO 12006-2 Building construction - Organization of information about construction works - Framework for classification of information, DIS version 2001

7. Lima, C., Fiès, B., Ferreira-da-Silva, C.: Setting up the Open Semantic Infrastructure for the Construction Sector in Europe - the FUNSIEC Project. In: 5th European Conference on Product and Process Modelling in the Building and Construction Industry - ECPPM 2004, Istambul, Turkey (2004).

8. Coghlan, D.: Practitioner research for organizational knowledge: Mechanistic and organistic-oriented approaches to insider action research. Management Learning, 34(4), (2003) 451-463.

9. Fricke, W. and Totterdill, P.: Action Research in Workplace Innovation and Regional Development. Amsterdam: John Benjamins (2004).

10.Reason, P.: Action research and the single case: A reply to Bjorn Gustavsen and Davydd Greenwood. Concepts and Transformation, 8(3), (2003) 281-294.

11.Avison, D., Lau, F., Myers, M. and Nielsen, P.: Action research. Communications of the ACM, 42(1), (1999) 94-97.

12.Coghlan, D.: Action research in the academy: Why and whither? Irish Journal of Management, (2006) 1-10.

13.Iversen, J., Mathiassen, L. and Nielsen, P.: Managing risk in software process improvement: an action research approach. MIS Quarterly, 28(3), (2004) 395-433.

14.Sense, A. Driving the bus from the rear passenger seat: Control dilemmas of participative action research. International Journal of Social Research Methodology, 9(1), (2006) 1-13.

15.Soares, A.L., Silva, M. Simões, D.: Selecting and structuring semantic resources to support SMEs, 8th International Conference on Enterprise Information Systems. Paphos, Cyprus. 2006. 